\title{
ORIGLALL PAPER
}

\section{Comparison of the Effect of Glutamate, Oxyferriscorbone and Deprenyl on Parkinsonian Syndrome}

\author{
Masharip Atadzhanov \\ Department of Medicine \\ University of Zambia School of Medicine and University Teaching Hospital \\ Lusaka, Zambia
}

\begin{abstract}
Background: Patients with Parkinson's disease (PD) show a dramatic increase in their brain iron content has suggested the role of iron in degeneration of dopaminergic nigrostriatal neurons in PD. Several studies have described the association of high dietary iron and PD. However, the role of iron the pathogenesis of PD is still hotly debated.
\end{abstract}

Objective: The purpose of this study was to investigate the effects of L-glutamate, oxyferriscorbone (OFC) and L-Deprenyl on parkinsonian syndrome (PS) in rats.

Methods: This study was performed on 244 male non-strain rats (250-270g;-10 wk old). By intranigral bilateral administration of 1-Methyl-4phenylpyridinium ion (MPP+) $(10 \mu \mathrm{g} / 2 \mu / 1$ into each side) and 6-hydroxydopamine (6-OHDA) $(12 \mu \mathrm{g} / 3 \mu \mathrm{l}$, Sigma, into each side) in rats was induced the dopamine deficient model of PS (DDPS) and the cholinergic model (ChPS) in rats produced by intracaudate injection of acetylcholine $(5 \mu \mathrm{g} / 2 \mu \mathrm{l}$ each side ) with neostigmine $(1 \mu \mathrm{g} / 1 \mu \mathrm{l}$ each side). These models were then used to investigate the effects of L-glutamate, OFC and L-Deprenyl on PS and the electric activity (EA) of the sensorimotor cortex, caudate nuclei, ventrolateral nuclei of the thalami, and substantia nigra in rats.

\section{Corresponding Author:}

Masharip Atadzhanov

Department of Medicine

University of Zambia, School of Medicine

Lusaka, Zambia
Results: Intracaudate injection of L-glutamate $(100 \mu \mathrm{g} / 3 \mu \mathrm{l}$, into each side) enhanced bradykinesia, rigidity and produced a weak tremor in the DDPS. This effect was more pronounced in ageing (12 months) rats and in some of them, we observed orofacial dyskinesia. In the ChPS, L-glutamate enhanced tremor and bradykinesia. The single and repeated injection of OFC $(5 ; 7 ; 5 ; 15$ and $20 \mathrm{mg} / \mathrm{rat}$, intraperitioneally, i.p.) did not produce a statistically significant change of PS in both models. L-deprenyl $(5,10$ and $20 \mathrm{mg} / \mathrm{kg}$, i.p.) dose-dependently reduced bradykinesia and rigidity in the DDPS, whereas increased tremor and failed to decrease bradykinesia and rigidity in the ChPS.

Conclusion: This study indicates that glutamate aggravates PS in both models. OFC does not have any effect on PS. Deprenyl has antiparkinsonian effect in the DDPS, but not in the ChPS.

\section{INTRODUCTION}

Parkinson's disease (PD) is a chronic progressive neurodegenerative disorder characterized by a loss of dopamine-producing neurons in the substantia nigra pars compacta (SNpc). The four cardinal motor symptoms of PD are resting tremor, bradykinesia (slowness), rigidity (muscle stiffness) and postural instability. A factor which triggers degenerative process is not known, so far. The deterioration and death of dopaminergic neurons are directly associated with misfolding and aggregation

Key words: Model of parkinsonism; glutamate, deprenyl, oxyferriscorbone Nigrostriatal system 
of proteins, principally alpha-synuclein, that are natively unfolded ${ }^{1}$. A central role of iron in the pathogenesis of PD has been emphasized because of its capacity to enhance the production of oxygen radicals and accelerate neuronal degeneration ${ }^{2}$. Several studies have suggested that increased iron content in the SN of patients with PD, even prior to the development of the disease symptoms ${ }^{3}$. A marked accumulation of iron related to disease severity is reported in the parkinsonian $\mathrm{SN}^{4}$. Iron has also been recently been implicated in promotion of alpha-synuclein aggregation either directly or via increasing levels of oxidative stress. Some studies have proposed the possible role of dietary iron in the development of PD. In recent study PD risk was significantly increased among individuals with high non-heme iron and low vitamin $C$ intake ${ }^{5}$. Presently it is still hotly debated causative role of the cellular iron dysregulation in $\mathrm{PD}^{6}$.

The activity of transmitter glutamate is most likely altered in the striatum in patients with PD. The role of glutamate in the N-methyl-4phenyldihydrpyridinium (MPP+) toxicity has been argued in the past decade. Monoamine oxidase type B (MAO-B) inhibitors have been used as symptomatic therapy for PD for approximately 20 years. The effect of L-Deprenyl (Selegeline $\mathrm{HCl}$ ), relatively selective irreversible MAO-B inhibitor, in treatment of PD is well established. At present it is known that L-Deprenyl has distinct MAOBindependent effects, and its neuroprotective effect is not correlated with MAOB inhibitor effect ${ }^{7,8}$.

The results of our previous investigations and literature data allowed us to classify animal models of Parkinsonism (P) as "dopamine deficient" or "cholinergic". The dopamine-deficient model is related primarily to reduced striatal dopamine (DA) and cholinergic model is linked to hyperactivity of cholinergic neurons in the caudate nuclei (CD). Based on our studies we proposed the existence of two forms of PD. In one, the pathologic changes begin in the $\mathrm{SN}$ or caudate nuclei, as a result of primary DA deficiency in the nigrostriatal system, and it is manifested by bradykinesia and rigidity dominated form of the disease. In the second, the process begins with primary activation of cholinergic neurons in the caudate nuclei, and is manifested by a tremor dominated form ofPD.
The purpose of this study was to investigate the effects of L-glutamate, oxyferriscorbone and Ldeprenyl on parkinsonian syndrome (PS) in dopamine deficient and cholinegic models of PS in rats.

\section{MATERIALAND METHODS}

\section{Animals and models of the parkinsonian syndrome}

This study was performed on 244 male non-strain rats $(250-270 \mathrm{~g} ;-10 \mathrm{wk}$ old), grown in our animal breeding facility. The animals were housed in a temperature controlled room with free access to food and water and were maintained in $12 \mathrm{~h}$ light/dark cycles. The dopamine deficient model of PS (DDPS) was induced by stereotactically and bilaterally intranigral administration of 1-Methyl4-phenylpyridinium ion (MPP+) $(10 \mu \mathrm{g} / 2 \mu / 1$ into each side) and 6-hydroxydopamine (6-OHDA) $(12 \mu \mathrm{g} / 3 \mu \mathrm{l}$, Sigma, into each side ) and the cholinergic model (ChPS) produced by intracaudate injection of acetyl-choline $(5 \mu \mathrm{g} / 2 \mu \mathrm{l}$ each side ) with neostigmine $(1 \mu \mathrm{g} / 1 \mu \mathrm{l}$ each side). Control rats that similarly received saline solution. The development of extrapyramidal disorders was studied simultaneously with EEG-recording. The electric activity (EA) was recorded in the sensorimotor cortex (SC), caudate nuclei (CD), ventrolateral nuclei of the thalami (TH), and substantia nigra (SN). Method of registration of the brain EA and evaluation of extrapyramidal symptoms (tremor, rigidity and bradykinesia described earlier ${ }^{10}$.

\section{Pharmacological analysis}

22 experimental groups were constructed with 12 rats in each group. Glutamate, oxyferriscorbone (OFC) and L-Deprenyl were administrated when PS was manifested significantly. The rats in groups 1-11 had MPP+ induced PS: in group 1 were injected only once intracaudate L-glutamate (sigma Chemical Co.,) one dose $100 \mu \mathrm{g} / 3 \mu \mathrm{l}$ into each side and in control group 2 were injected the same volume of normal saline; in groups 3,4,5,6,7 animals received once intraperitoneally (i.p) different doses of OFC (group 3 received $5 \mathrm{mg}$ in 1 $\mathrm{ml}$ normal saline, group $4-7.5 \mathrm{mg} / \mathrm{ml}$, group5- 
$15 \mathrm{mg} / \mathrm{ml}$, group $6-20 \mathrm{mg} / \mathrm{ml}$ of OFC and in group 7 control rats received i.p. $1 \mathrm{ml}$ normal saline. In groups 8-10 animals received i.p. one different dose of L-deprenyl (CHINOIN Pharmaceutical and Chemical products Co) $(5,10$ and $20 \mathrm{mg} / \mathrm{kg}$, respectively) in $1 \mathrm{ml}$ volume. In group 11 control rats received i.p. $1 \mathrm{ml}$ normal saline. Animals in groups 12-22 had acetylcholine-induced PS and had been administrated L-glutamate, OFC and Ldeprenyl in the same regime as rats in 1-11 groups. Motor functions of rats were measured 8 hours after drugs injections and EA was recorded every day for 2 weeks.

\section{Statistics}

Means and standard errors of the means (SEM) were calculated for each series of experiments. Student's t-test was used to assess the significance of differences between the different groups of experiments. Values of $\mathrm{P}$ less than 0.05 were considered significant.

\section{RESULTS}

Glutamate significantly enhanced bradykinesia, rigidity and produced a weak tremor in the dopamine deficient model of PS. The effect of glutamate was observed from 3 to 6 hours after injection (Fig. $1 \mathrm{~A}$ ). This effect was more marked in ageing (15 months) animals and in some of them registered orofacial dyskinesias (results not shown) In the cholinergic model glutamate enhanced tremor and hypokinesia. The effect of glutamate on hypokinesia lasted for 6 hours while its effect on tremor lasted for 2 to 3 hours after injection (Fig. 1B). Administration of glutamate dramatically changed EA of the SC, $\mathrm{CN}$ and $\mathrm{SN}$ in MPP+induced PS. In all the brain structures investigated, a high amplitude paroxysmal activity (PA) of "peakwave" type were registered. This -remained steady (or persisted) for 2 hours after injection of glutamate (Fig.2). Repeated intraperitoneal administration of various doses of oxyferriscorbone did not produce a statistically significant change of extrapyramidal symptoms in both models of PS (Fig.3). OFC did not have any effects on EA of the investigated brain structures.

L-deprenyl dose- dependently reduced bradykinesia and rigidity in the DDPS. This effects lasted for 2 to 3 hours. Reduction of extrapyramidal symptoms under deprenyl was followed by suppression of the high amplitude PA in the SC and subcortical structures

(Fig. 5). Deprenyl did not produce any noticeable effect on severity of tremor but prolonged its duration in the cholinergic model of parkinsonian syndrome (Fig. 4)

\section{DISCUSSION}

The present results demonstrate that intrastriatal glutamate injection increased PS symptoms induced both by dopamine deficiency in the striatum and hyperactivation of cholinergic neurons in the caudate nuclei. The severity of parkinsonian syndrome after glutamate injection was agedependent. Such effect of glutamate very likely was connected with stimulation release of acetylcholine and inhibition release of endogenous dopamine in the striatum ${ }^{11,12}$. According to SA Lipton and PA Rosenberg ${ }^{13}$ glutamate may exert damaging effect on neurons and lead to death of the cells. On the basis of above data we may conclude that drugs which primarily activate the glutamatergic system in the striatum should not be recommended for treating patients with Parkinsonism.

There are a number of data about an important role of iron metabolism in the basal ganglia in the pathogenesis of neurodegenerative disorders, including $\mathrm{PD}^{2,4}$. In addition, it is supposed to be a neuroprotective effect of iron chelaters ${ }^{14}$, and beneficial effect of iron in patients with $\mathrm{PD}^{15}$. However, results of the present study are in contrast to the reports about clinical effect of iron in Parkinson patients. We could not register any improvement of hypokinesia, rigidity and tremor neither in the dopamine deficient model nor in the cholinergic model of PS. Therefore, we may suggest that iron containing drugs cannot exert principal influence on the strategy of treatment of patients with Parkinsonism.

Our study has demonstrated unequivocal beneficial effect of deprenyl in the DDPS, in which it decreased hypokinesia and rigidity. However, deprenyl increased tremor and failed to decrease hypokinesia and rigidity in the cholinergic model of 
PS. It is possible, based on present data; deprenyl does not influence the cholinergic system in the brain or symptoms caused by primary hyperactivation of this system. Some studies ${ }^{16,17}$ suggest that deprenyl may influence the rate of neuronal degeneration through mechanisms that are not dependent on MAOB inhibition. Nevertheless, we propose that clinical effect of deprenyl in PD patients is mainly connected with its influence on dopamine metabolism in the basal ganglia. Glutamate aggravates parkinsonian syndrome in both models, deprenyl has antiparkinsonian effect in the dopamine deficient model but not in the cholinergic model of parkinsonian syndrome. In both models, oxyferriscorbone does not have any effect on PS.

The role of iron in neurodegeneration is controversial and still under debate. The increased ferritin and iron and loss of glutathione have prompted the hypothesis of free ionic iron participating in oxidative stress initiated dopamine neuronal death in substantia nigra ${ }^{18}$. The role of iron in neurodegeneration is supported by recent reports that nutritional iron deficiency in rats, which reduces brain iron and alters iron regulatory proteins $^{19}$, prevents the neurotoxicity of 6 hydroxydopamine and kainite ${ }^{20}$.These authors suggest aetiopathogenic role of iron in PD. Whilst others $^{21}$ recognizing the role of iron in oxidative stress, which plays a major role in neurodegeneration in PD, consider the hypothesis that iron might drive PD neurodegeneration was postulated when our understanding of PD molecular pathophysiology was far more rudimentary. Several lines of current investigation do not support an "upstream" role for iron in PD neurodegeneration ${ }^{22}$. The observation that iron concentrations are relatively high in the substantia nigra and even more so in PD are not sufficient to establish an important causal role for iron in $\mathrm{PD}^{21}$.

The results of our experiments support this opinion and suggest that the development of the management of patients with PD with iron containing drugs is not perspective.

\section{ACKNOWLEDGMENT}

The author would like to thank Prof. G.N. Kryzhanovsky and all staff of the laboratory of pathology of the Central Nervous System, Research Institute of General Pathology and Pathophysiology, Academy of Medical Sciences of the Russian Federation, for their assistance and support.

\section{REFERENCES}

1. Arreguin S, Nelson P, Padway S, et al. Dopamine complexes of iron in the aetiology and pathogenesis of Parkinson's disease. J Inorg Biochem 2009; 103(1):87-93.

2. Berg D, Gerlach M, Youdim MBH, et al. Brain iron pathways and their relevance to Parkinson's disease. JNeurochem 2001; 79:225-236.

3. Becker G, Seufert J, Bogdahn U, et al. Degeneration of substantia nigra in chronic Parkinson's disease visualized by transcranial sonography. Neurology 1995; 45:182-184.

4. Zecca L, Youdim MB, Riederer P, et al. Iron, brain aging and neurodegenerative disorders. Nat Rev Neurosci 2004; 5(11):863-873.

5. Logroscino G, Gao X, Chen H, et al. Dietary intake and risk of Parkinson's disease. Am J Epidem 2008; 168(12):1381-1388.

6. Kaur D, Andersen J. Does cellular iron dysregulation play a causative role in Parkinson's disease? Ageing Res Rev 2004; 3(3):327-343.

7. Schapiro AHV, Olanow CW. Neuroprotection in Parkinson's disease: myths, mysteries and misconceptions. JAMA 2004; 291:358-364.

8. Marras C, McDermott MP, Rochon PA, et al.: Parkinson Study Group. Survival in Parkinson's disease: thirteen-year follow-up of the DATATOP cohort. Neurology 2005; 64:87-93.

9. Atadzhanov M, Rahimdzhanov A. Dopamine deficiency and cholinergic models of the parkinsonian syndrome. Neurology, 1992; 43; Suppl. 1:26-28.

10. Kryzhanovsky G.N, Atadzhanov M.A., Vorinian TA, Nerobkova LN. Characteristics of Parkinsonism induced by MPTP and stimulation of caudate nuclei cholinergic neurons, J Neural Psychiatr. (Rus), 1993: 6; 3-6. 
11. Kryzhanovsky G.N. The determinant structures and the pathology of the Nervous System, New York, 1986, 348p.

12. Bibbiani F, Oh JD, Kielaite A, et al. Combined blockade of AMPA and NMDA glutamate receptors in animal models of PD. Exp Neurol 2005; 196:422-429.

13. Lipton SD, Rosenberg PA. Mechanisms of disease: excitatory amino acid as a final common pathway for neurological disorders New EnglJMed, 1994; 330:613-622.

14. Shindou T, Shinch K, Kahaoka Y. Inhibitor effect of NMDA on dopaminergic transmission in a slice preparation of rat globus pallidus. Jap J Pharmacol, 1992; 58:79-82.

15. Birkmayer W, Birkmayer JGD. Iron, a new aid in the treatment of Parkinson patients. J Neural Transm, 1986; 67:287-292.

16. Tatton WG, Chalmers-Redman R, Tatton N. Neuroprotection by deprenyl and other propargylamines. J Neural Transm 2003; 110:509-515.

17. Wu RM, Chieuh CC, Perr A, Murphy DL. Apparent antioxidant effect of L-deprenylon hydroxyl radical formation and nigral injury elicited by MPP+ in vivo. Eur J Pharmacol, 1993; 243:241-247.
18. Olanow CW, Stern MB, Sethi K. The scientific and clinical basis for the treatment of Parkinson disease. Neurology 2009; 72(21) suppl 4 S1-S136.

19. Siddappa AJ, Rao RB, Wobken JD, et al. Iron deficiency alters iron regulatory protein and iron-transport protein expression in the rat brain. Pediatr Rws. 2003; 53:800-807.

20. Shoham S and Youdim MBH. Nutritional Iron deprivation attenuates kainite-induced neurotoxicity in rats: Implications for involvement of iron in neurodegeneration. Ann NYAcad Sci 2004; 1012:306.

21. Swerdlow RH. Iron plays a limited role in neurodegeneration. Moving Along. Spring 2004; 7 .

22. Dawson TM, Dawson VL. Molecular pathways of neurodegeneration in Parkinson's disease. Science 2003; 302:819-822.
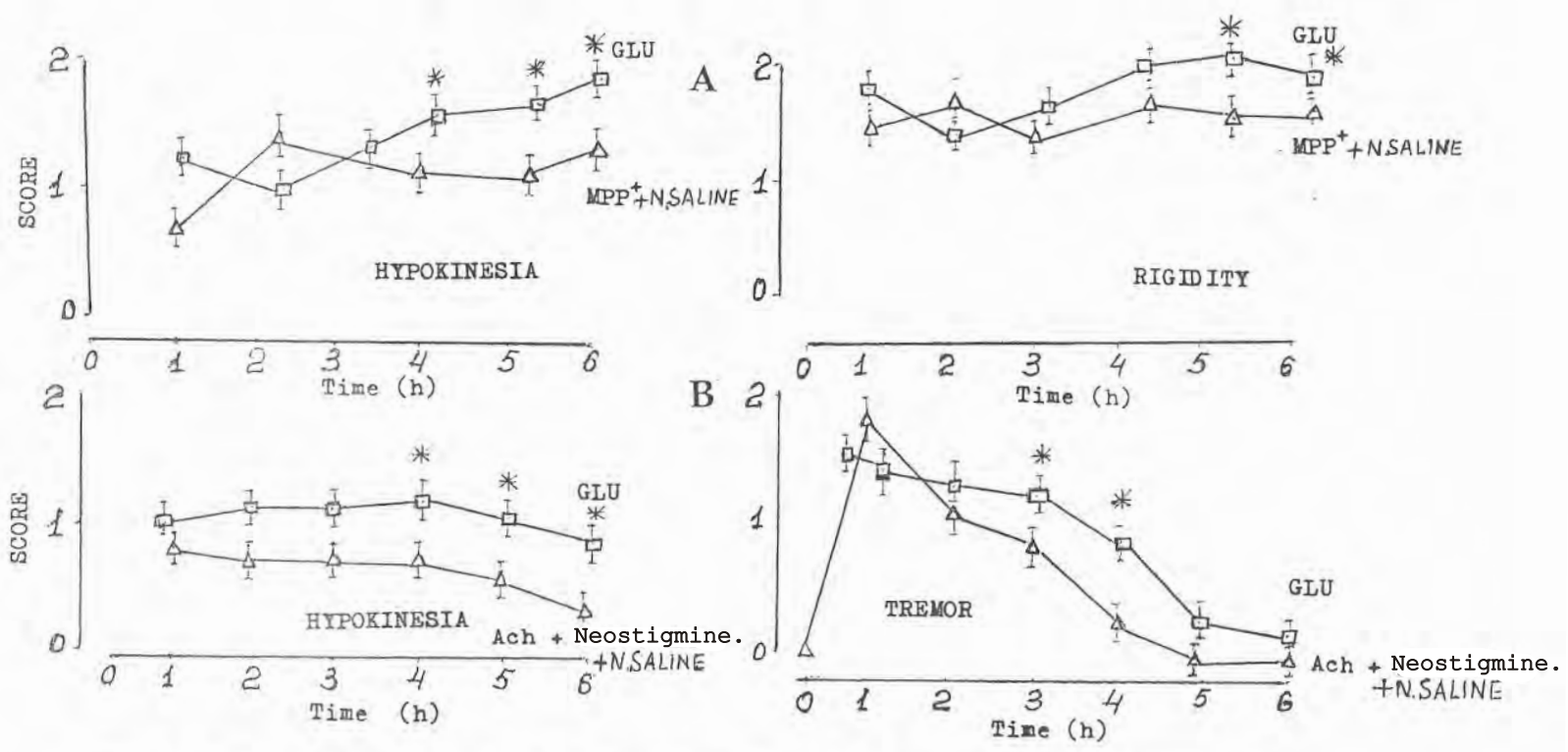

FIG I. EFFECTS OF GLUTAMATE ON DOPAMINE-DEFICIENT (A) AND CHOLINERGIC MODELS (B)

OF PARKINSONIAN SYNDROME IN RATS.

* $P<0,05$ 


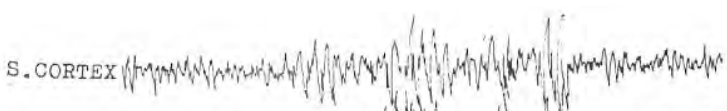

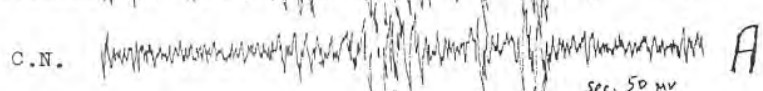

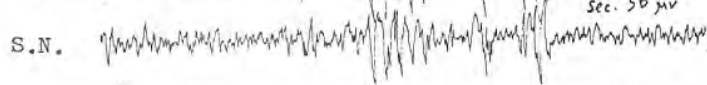

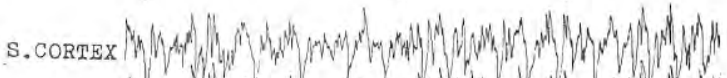

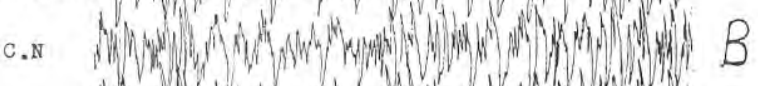

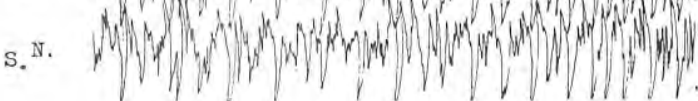

Fig. 2. ELECTRICAL ACTIVITY OF SENSORIMOTOR CORTEX, CAUD ATE NUCLEI (C.N.), ANע SUBSTANTIA NIGRA (S.N.) AFTER $48 \mathrm{hs}$ INTRANIGRAL INJECTION OF MPP ${ }^{+}$(A) AND

$4 \mathrm{hs}$ AFTER INTRACAUd ATE INJECTION OF GLUTAMATE(B).

\section{A}

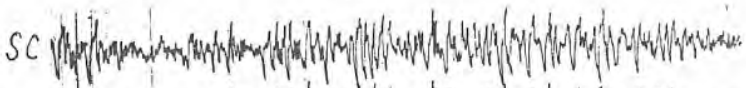

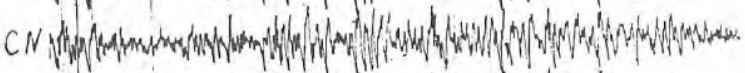

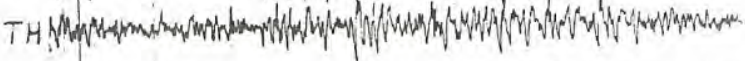

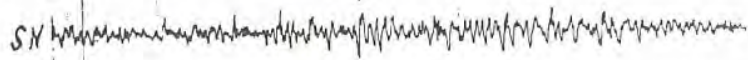
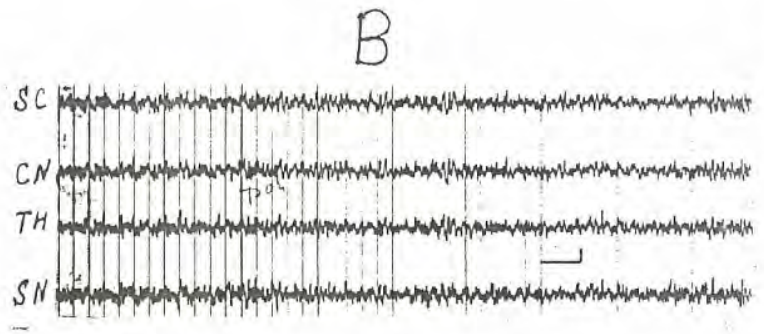

FIG.5. ELECTRICAL ACTIVITY OF SENSORIMOTOR CORTEX (SC), CAUDATE NUCLEI (CN), THALAMUS (TH) AND SUBSTANTIA NIGRA (SN) AFTER $48 \mathrm{hs}$ OF MPP ${ }^{+}$(A) AND 4 hS AFTER ADMINISTRATION QF DEPRENYI (B).

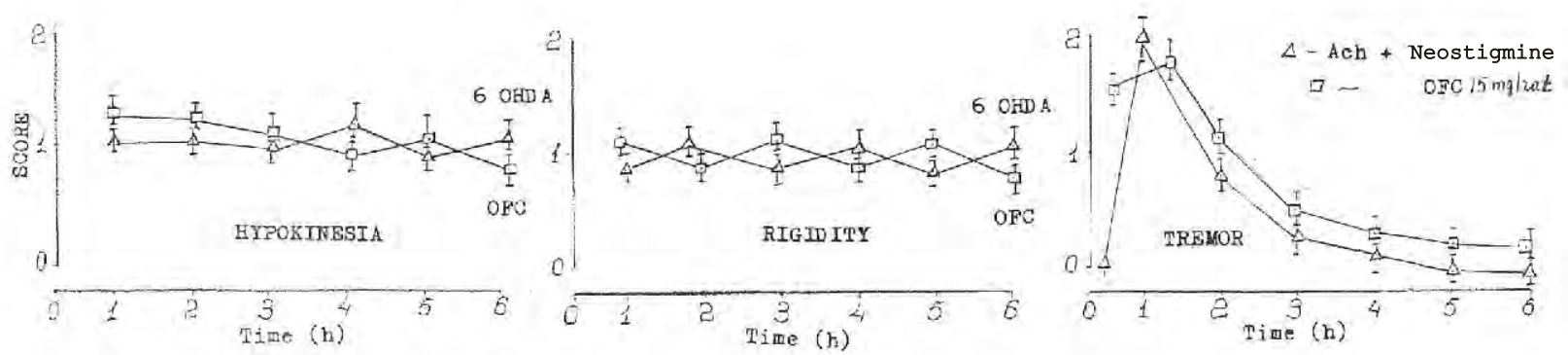

PIG.3. BEFECHS OE REPEATED AD WIMISTRATION OF OXYFERRISCORBONE ON PARKINSONIAN SYNDROME TNDJCZE BE 5 OHD A AND ACEITLCHOLINZ (ACh) WITH NEOSTIGMINE.
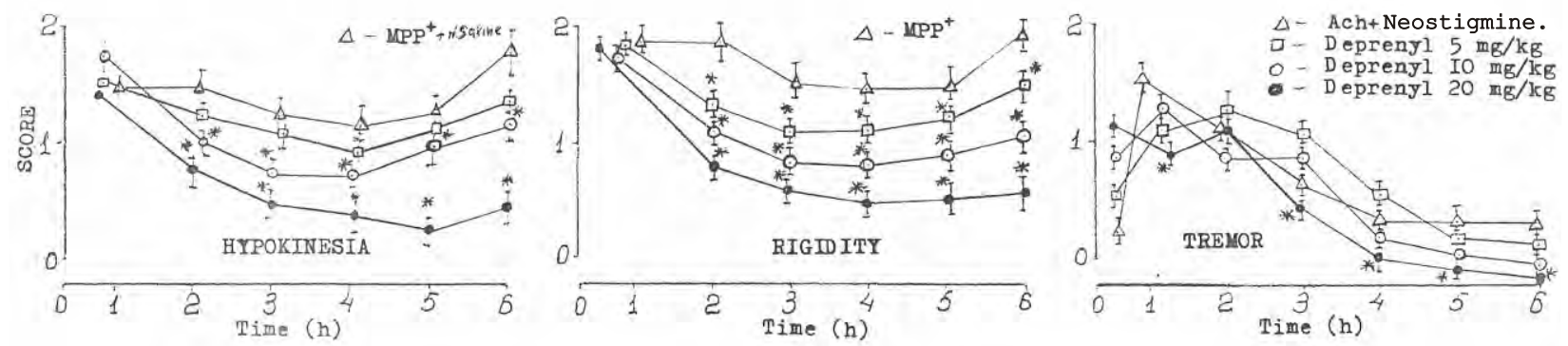

FIG. 4. EFFECTS OF VARIOUS DOSES OF DEPRENYL ON PARKINSONIAN SYNDROME INDUCED BY INTRANIGRAL INJECTION OF MPP ${ }^{+}$AND ACETYLCHOLINE WITH NEOSTIGMINE.

* $\quad P<0,05$ 